Internat. J. Math. \& Math. Sci.

Vol. 24, No. 4 (2000) 217-230

S0161171200004737

(C) Hindawi Publishing Corp.

\title{
PERISTALTIC TRANSPORT IN A CYLINDRICAL TUBE THROUGH A POROUS MEDIUM
}

\author{
ELSAYED F. EL SHEHAWEY and WAHED EL SEBAEI
}

(Received 1 March 2000)

\begin{abstract}
The problem of peristaltic transport in a cylindrical tube through a porous medium has been investigated. A perturbation solution is obtained, which satisfies the momentum equation for the case in which the amplitude ratio is small. The results show that the fluid phase mean axial velocity increases with increasing the permeability parameter $k$. The phenomena of reflux is discussed. Numerical results are reported for various values of the physical parameter.
\end{abstract}

Keywords and phrases. Peristaltic transport, porous medium.

2000 Mathematics Subject Classification. Primary 76Z05, 76S05.

1. Introduction. The word peristaltic stems from the Greek word peristaltikos, which means clasping and compressing. It occurs due to the action of a progressive waves which propagates along the length of a distensible tube containing liquid. Peristaltic pumping has been the object of scientific and engineering researchs during the recent past few decades.

The pumping of fluids through muscular tubes by means of peristaltic waves is an important biological mechanism. In particular, peristaltic mechanism may be involved in swallowing food through the oesophagus, urine transport from kidney to bladder through the ureter.

Study of the mechanism of peristalsis from both the mechanical and physiological view points has been the object of scientific research. Since the first investigation of Latham [5], several theoretical and experimental attempts have been made to understand peristaltic action in different situations. A review of much of the early literature is presented by Jaffrin and Shapiro [4]. A summary of most of the experimented and theoretical investigations reported with details of the geometry, fluid, Reynolds number, wavelength parameter, wave amplitude parameter, and wave shape have been given by Srivastava and Srivastava [13]. Srivastava and Srivastava [14] studied the effects of Poiseuille flow on peristaltic transport of a particulate suspension. Recently Saxena and Srivastava [11] studied the particulate suspension flow induced by sinusoidal peristaltic waves. In another type of studies on peristaltic transport, El Misery, El Shehawey, and Hakkem [1] studied the peristaltic motion of an incompressible generalized Newtonian fluid in a planer channel. Mekheimer, El Shehawey, and Elaw [6] studied the peristaltic motion of a particle fluid suspension in a planer channel. Flow through a porous medium has been of considerable interest in recent years particularly among geophysical fluid dynamicists. Many technical processes involve parallel 
flow of fluids of different viscosity and density through porous media. Such parallel flows exist in packed bed reactors in the chemical industry, in petroleum production engineering, and in many other processes as well. Flows through porous medium occur in filtration of fluids and seepage of water in river beds. Movement of underground, water and oils, beach sand stand stone, limestone, rye bread, wood, the human lung, bile duct, gall bladder with stones, and small blood vessels are some important examples of flow through porous medium. Another example is the seepage under a dam which is very important [10]. Several works have been published by using the generalized Darcy's law [12], where the convection acceleration and viscous-stress are taken into account [16].

El Sayed [2] studied the electrohydrodynamic instability of two superposed viscous and streaming fluids through porous medium. Varshney [15] studied the fluctuating flow of a viscous fluid through a porous medium bounded by porous and horizontal surface. Raptis et al. [8, 9] studied the steady free convection and mass transfer flow of a viscous fluid through a porous media bounded by a vertical surface. El Shehawey et al. [3] studied the peristaltic transport through a porous medium. In the case of high permeability parameter (as $k \rightarrow \infty$ ), our result is in agreement with Yih and Fung [17].

2. Formulation of the problem. We shall consider a two-dimensional circular cylindrical tube of radius $R$, with axisymmetric, moderate-amplitude traveling waves imposed on its wall is considered.

The fluid is assumed to be Newtonian, viscous, homogeneous, and incompressible through a porous medium occupying a semi-infinite region of the space. The equations governing two-dimensional motion of a viscous incompressible fluid through a porous medium are (see [7])

$$
\begin{aligned}
& \frac{\partial v_{r}}{\partial t}+v_{r} \frac{\partial v_{r}}{\partial r}+v_{z} \frac{\partial v_{r}}{\partial z}=-\frac{1}{\rho} \frac{\partial P}{\partial r}+v\left(\frac{\partial^{2} v_{r}}{\partial r^{2}}+\frac{\partial^{2} v_{r}}{\partial z^{2}}+\frac{1}{r} \frac{\partial v_{r}}{\partial r}-\frac{v_{r}}{r^{2}}\right)-\frac{v}{k} v_{r} \\
& \frac{\partial v_{z}}{\partial t}+v_{r} \frac{\partial v_{z}}{\partial r}+v_{z} \frac{\partial v_{z}}{\partial z}=-\frac{1}{\rho} \frac{\partial P}{\partial z}+v\left(\frac{\partial^{2} v_{z}}{\partial r^{2}}+\frac{\partial^{2} v_{z}}{\partial z^{2}}+\frac{1}{r} \frac{\partial v_{z}}{\partial r}\right)-\frac{v}{k} v_{z}
\end{aligned}
$$

and the equation of continuity is

$$
\frac{\partial v_{r}}{\partial r}+\frac{\partial v_{z}}{\partial z}+\frac{v_{r}}{r}=0
$$

where $z$ is the axial coordinate in the direction of wave propagation, $r$ is the radial coordinate, $v$ is kinematic viscosity, $\rho$ is the density, $P$ is the pressure, $t$ is the time, $v_{r}$ and $v_{z}$ are velocity components in the $r$ - and $z$-directions, respectively, and $k$ is the permeability parameter. Axisymmetric motion is assumed.

The velocity components can be written in terms of Stoke's stream function $\Psi$ as

$$
v_{r}=\frac{1}{r} \frac{\partial \Psi}{\partial z}, \quad v_{z}=-\frac{1}{r} \frac{\partial \Psi}{\partial r}
$$




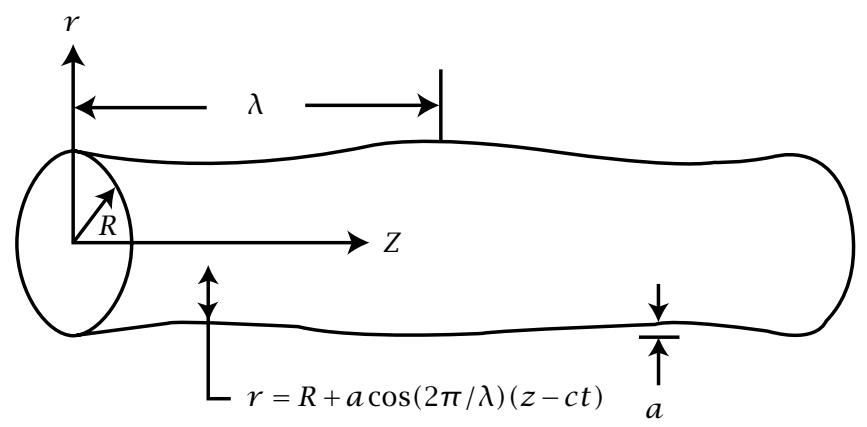

FIGURE 2.1. Geometry of the problem.

Eliminating pressure $P$ between equations (2.1a) and (2.1b) using equation (2.3) yields the equation for the stream function,

$$
\frac{\partial}{\partial t}\left(\bar{\nabla}^{2} \Psi\right)+\frac{1}{r} \frac{\partial \Psi}{\partial z}\left[\bar{\nabla}^{2}\left(\frac{\partial \Psi}{\partial r}\right)-\frac{2}{r} \bar{\nabla}^{2} \Psi+\frac{1}{r^{2}} \frac{\partial \Psi}{\partial r}\right]-\frac{1}{r} \frac{\partial \Psi}{\partial r} \bar{\nabla}^{2}\left(\frac{\partial \Psi}{\partial z}\right)=\nu \bar{\nabla}^{2} \bar{\nabla}^{2} \Psi-\frac{v}{k} \bar{\nabla}^{2} \Psi,
$$

where $\bar{\nabla}^{2}$ is a special operator defined as

$$
\bar{\nabla}^{2}=\frac{\partial^{2}}{\partial z^{2}}+\frac{\partial^{2}}{\partial r^{2}}-\frac{1}{r} \frac{\partial}{\partial r} .
$$

At the boundaries, the fluid is subjected to the motion of the wall in the form (see Figure 2.1),

$$
\eta=a \cos \frac{2 \pi}{\lambda}(z-c t),
$$

where $a$ is the amplitude, $\eta$ is the radial displacement from the mean position of the wall, $\lambda$ is the wavelength, and $c$ is the wave speed. The boundary conditions that must be satisfied by the fluid on the walls are the no-slip and impermeability conditions. The velocity components of the fluid particles on the wall are thus

$$
v_{z}=0, \quad v_{r}=\frac{\partial \eta}{\partial t}
$$

and in terms of the stream function $\Psi$,

$$
\frac{\partial \Psi}{\partial r}=0, \quad \frac{\partial \Psi}{\partial z}=\frac{2 \pi a c r}{\lambda} \sin \frac{2 \pi}{\lambda}(z-c t),
$$

we introduce the non-dimensional variables and parameters as follows:

$$
\begin{gathered}
r^{*}=\frac{r}{R}, \quad z^{*}=\frac{z}{R}, \quad v_{r}^{*}=\frac{v_{r}}{c}, \quad v_{z}^{*}=\frac{v_{z}}{c}, \quad \eta^{*}=\frac{\eta}{R}, \\
\Psi^{*}=\frac{\Psi}{R^{2} c}, \quad t^{*}=\frac{c t}{R}, \quad P^{*}=\frac{p}{\rho c^{2}}, \quad k^{*}=\frac{k}{R^{2}},
\end{gathered}
$$


and the amplitude ratio $\epsilon$, the wave number $\alpha$, and the Reynolds number Re. These parameters are defined as:

$$
\epsilon=\frac{a}{R}, \quad \alpha=\frac{2 \pi R}{\lambda}, \quad \operatorname{Re}=\frac{c R}{v} .
$$

The non-dimensional forms of (2.4), (2.6), and (2.8), after dropping the star are:

$$
\begin{gathered}
\frac{\partial}{\partial t}\left(\bar{\nabla}^{2} \Psi\right)+\frac{1}{r} \frac{\partial \Psi}{\partial z}\left[\bar{\nabla}^{2} \frac{\partial \Psi}{\partial r}-\frac{2}{r} \bar{\nabla}^{2} \Psi+\frac{1}{r^{2}} \frac{\partial \Psi}{\partial r}\right]-\frac{1}{r} \frac{\partial \Psi}{\partial r} \bar{\nabla}^{2} \frac{\partial \Psi}{\partial z}=\frac{1}{\operatorname{Re}} \bar{\nabla}^{2}\left(\bar{\nabla}^{2}-\frac{1}{k}\right) \Psi, \\
\eta=\epsilon \cos \alpha(z-t), \\
\frac{\partial \Psi}{\partial r}=0, \quad \frac{\partial \Psi}{\partial z}=\alpha \epsilon r \sin \alpha(z-t), \quad r=1+\eta
\end{gathered}
$$

3. Method of solution. We expand $\Psi$ and $\partial P / \partial z$ in a power series of the small parameter $\epsilon$,

$$
\begin{gathered}
\Psi=\Psi_{0}+\epsilon \Psi_{1}+\epsilon^{2} \Psi_{2}+\cdots \\
\frac{\partial P}{\partial z}=\left(\frac{\partial P}{\partial z}\right)_{0}+\epsilon\left(\frac{\partial P}{\partial z}\right)_{1}+\epsilon^{2}\left(\frac{\partial P}{\partial z}\right)_{2}+\cdots
\end{gathered}
$$

On substituting (3.1) into (2.10), collecting terms of equal powers of $\epsilon$, and equating the coefficients of like powers on both sides of the equation, we obtain

$$
\begin{aligned}
& \frac{1}{\operatorname{Re}} \bar{\nabla}^{2}\left(\bar{\nabla}^{2}-\frac{1}{k}\right) \Psi_{0} \frac{\partial}{\partial t} \bar{\nabla}^{2} \Psi_{0}+\frac{1}{r} \frac{\partial \Psi_{0}}{\partial z}\left[\bar{\nabla}^{2} \frac{\partial \Psi_{0}}{\partial r}-\frac{2}{r} \bar{\nabla}^{2} \Psi_{0}+\frac{1}{r^{2}} \frac{\partial \Psi_{0}}{\partial r}\right]-\frac{1}{r} \frac{\partial \Psi_{0}}{\partial r} \bar{\nabla}^{2} \frac{\partial \Psi_{0}}{\partial z} \\
& \frac{1}{\operatorname{Re}} \bar{\nabla}^{2}\left(\bar{\nabla}^{2}-\frac{1}{k}\right) \Psi_{1} \\
&=\frac{\partial}{\partial t} \bar{\nabla}^{2} \Psi_{1}+\frac{1}{r} \frac{\partial \Psi_{1}}{\partial z}\left[\bar{\nabla}^{2} \frac{\partial \Psi_{0}}{\partial r}-\frac{2}{r} \bar{\nabla}^{2} \Psi_{0}+\frac{1}{r^{2}} \frac{\partial \Psi_{0}}{\partial r}\right] \\
&+\frac{1}{r} \frac{\partial \Psi_{0}}{\partial z}\left[\bar{\nabla}^{2} \frac{\partial \Psi_{1}}{\partial r}-\frac{2}{r} \bar{\nabla}^{2} \Psi_{1}+\frac{1}{r^{2}} \frac{\partial \Psi_{1}}{\partial r}\right]-\frac{1}{r} \frac{\partial \Psi_{1}}{\partial r} \bar{\nabla}^{2} \frac{\partial \Psi_{0}}{\partial z}-\frac{1}{r} \frac{\partial \Psi_{0}}{\partial r} \bar{\nabla}^{2} \frac{\partial \Psi_{1}}{\partial z}, \\
& \frac{1}{\operatorname{Re}} \bar{\nabla}^{2}\left(\bar{\nabla}^{2}-\frac{1}{k}\right) \Psi_{2} \\
&=\frac{\partial}{\partial t} \bar{\nabla}^{2} \Psi_{2}+\frac{1}{r} \frac{\partial \Psi_{2}}{\partial z}\left[\bar{\nabla}^{2} \frac{\partial \Psi_{0}}{\partial r}-\frac{2}{r} \bar{\nabla}^{2} \Psi_{0}+\frac{1}{r^{2}} \frac{\partial \Psi_{0}}{\partial r}\right] \\
&+\frac{1}{r} \frac{\partial \Psi_{1}}{\partial z}\left[\bar{\nabla}^{2} \frac{\partial \Psi_{1}}{\partial r}-\frac{2}{r} \bar{\nabla}^{2} \Psi_{1}+\frac{1}{r^{2}} \frac{\partial \Psi_{1}}{\partial r}\right]+\frac{1}{r} \frac{\partial \Psi_{0}}{\partial z}\left[\bar{\nabla}^{2} \frac{\partial \Psi_{2}}{\partial r}-\frac{2}{r} \bar{\nabla}^{2} \Psi_{2}+\frac{1}{r^{2}} \frac{\partial \Psi_{2}}{\partial r}\right] \\
&-\frac{1}{r} \frac{\partial \Psi_{2}}{\partial r} \bar{\nabla}^{2} \frac{\partial \Psi_{0}}{\partial z}-\frac{1}{r} \frac{\partial \Psi_{1}}{\partial r} \bar{\nabla}^{2} \frac{\partial \Psi_{1}}{\partial z}-\frac{1}{r} \frac{\partial \Psi_{0}}{\partial r} \bar{\nabla}^{2} \frac{\partial \Psi_{2}}{\partial z} .
\end{aligned}
$$


We expand the boundary conditions (2.12) in power series of $\eta$ :

$$
\begin{gathered}
\Psi_{r}(1)+\eta \Psi_{r r}(1)+\frac{\eta^{2}}{2} \Psi_{r r r}(1)+\cdots=0, \\
\Psi_{z}(1)+\eta \Psi_{r z}(1)+\frac{\eta^{2}}{2} \Psi_{z r r}(1)+\cdots=\alpha \epsilon \sin \alpha(z-t) .
\end{gathered}
$$

On substituting (2.11) and (3.1) into (3.6) and (3.7) and equating coefficients like powers of $\epsilon$ on both sides of the equation we obtain

$$
\begin{aligned}
& \frac{\partial \Psi_{0}}{\partial r}(1)=0 \\
& \frac{\partial \Psi_{1}}{\partial r}(1)+\frac{\partial^{2} \Psi_{0}}{\partial r^{2}}(1) \cos \alpha(z-t)=0 \\
& \frac{\partial \Psi_{2}}{\partial r}(1)+\frac{\partial^{2} \Psi_{1}}{\partial r^{2}}(1) \cos \alpha(z-t)+\frac{1}{2} \frac{\partial^{3} \Psi_{0}}{\partial r^{3}}(1) \cos ^{2} \alpha(z-t)=0, \\
& \frac{\partial \Psi_{0}}{\partial z}(1)=0, \\
& \frac{\partial \Psi_{1}}{\partial z}(1)+\frac{\partial^{2} \Psi_{0}}{\partial r \partial z}(1) \cos \alpha(z-t)=\alpha \sin \alpha(z-t), \\
& \frac{\partial \Psi_{2}}{\partial z}(1)+\frac{\partial^{2} \Psi_{1}}{\partial r \partial z}(1) \cos \alpha(z-t)+\frac{1}{2} \frac{\partial^{3} \Psi_{0}}{\partial r^{2} \partial z}(1) \cos ^{2} \alpha(z-t)=0 .
\end{aligned}
$$

Equations (3.3), (3.8), and (3.11), together with a condition of uniform pressure gradient $(\partial P / \partial z)_{0}=$ constant, are satisfied to yield the classical Poiseuille flow for a fluid through a porous medium

$$
\Psi_{0 r}=\bar{k}\left(I_{0}\left(\frac{1}{\sqrt{k}}\right) r-I_{0}\left(\frac{1}{\sqrt{k}} r\right) r\right),
$$

where

$$
\bar{k}=\frac{\operatorname{Re} k}{I_{0}(1 / \sqrt{k})}\left(\frac{\partial P}{\partial z}\right)_{0}
$$

is the Poiseuille flow parameter for a fluid through a porous medium, $I_{0}$ and $I_{1}$ are the modified Bessel functions of the first kind.

Equation (3.4) with the corresponding boundary conditions (3.9) and (3.12) shows that a solution can be chosen in the form

$$
\Psi_{1}=\phi_{1}(r) e^{i \alpha(z-t)}+\phi_{1}^{*}(r) e^{-i \alpha(z-t)}
$$

where the asterisk denote a complex conjugate.

On substituting (3.16) into (3.4), (3.9), and (3.12), we obtain the Orr-Sommerfeld equation with the corresponding boundary conditions as follows:

$$
\begin{aligned}
{\left[\frac{d^{2}}{d r^{2}}-\frac{1}{r} \frac{d}{d r}\right.} & \left.-\alpha^{2}+\frac{1}{k}+i \alpha \operatorname{Re}\left[1+\bar{k}\left(I_{0}\left(\frac{1}{\sqrt{k}}\right)-I_{0}\left(\frac{r}{\sqrt{k}}\right)\right)\right]\right] \\
\times & {\left[\frac{d^{2}}{d r^{2}}-\frac{1}{r} \frac{d}{d r}-\alpha^{2}\right] \phi_{1}+\frac{i \alpha \operatorname{Re} \bar{k}}{r}\left(\frac{1}{k} I_{0}\left(\frac{r}{\sqrt{k}}\right) r-\frac{2}{\sqrt{k}} I_{1}\left(\frac{r}{\sqrt{k}}\right)\right) \phi_{1}=0, }
\end{aligned}
$$




$$
\phi_{1}^{\prime}(1)=\frac{\bar{k}}{2 \sqrt{k}} I_{1}\left(\frac{1}{\sqrt{k}}\right), \quad \phi_{1}(1)=-\frac{1}{2},
$$

where the primes denote differentiation with respect to $r$.

Equation (3.5) with the corresponding boundary conditions (3.10) and (3.13) shows that a solution can be chosen in the form

$$
\Psi_{2}=\phi_{20}(r)+\phi_{22}(r) e^{2 i \alpha(z-t)}+\phi_{22}^{*}(r) e^{-2 i \alpha(z-t)} .
$$

On substituting (3.19) into (3.5), (3.10), and (3.13), we obtain the two equations for $\phi_{20}$ and $\phi_{22}$ with the corresponding boundary conditions as follows:

$$
\begin{aligned}
& \overline{\bar{\nabla}}^{2}\left(\overline{\bar{\nabla}}^{2}-\frac{1}{k}\right) \phi_{20} \\
&= \frac{i \alpha \operatorname{Re}}{r}\left[-\frac{3}{r} \phi_{1} \phi_{1}^{*^{\prime \prime}}+\phi_{1} \phi_{1}^{*^{\prime \prime \prime}}+\frac{3}{r^{2}} \phi_{1} \phi_{1}^{*^{\prime}}+\frac{3}{r} \phi_{1}^{\prime \prime} \phi_{1}^{*}\right. \\
&\left.\quad-\phi_{1}^{*} \phi_{1}^{\prime \prime \prime}-\frac{3}{r^{2}} \phi_{1}^{\prime} \phi_{1}^{*}+\phi_{1}^{\prime} \phi_{1}^{*^{\prime \prime}}-\phi_{1}^{*^{\prime}} \phi_{1}^{\prime \prime}\right], \\
& \tilde{\nabla}^{2}\left(\tilde{\tilde{\nabla}}^{2}-\frac{1}{k}\right) \phi_{22} \\
&=-2 i \alpha \operatorname{Re}\left[1-i \bar{k}\left(I_{0}\left(\frac{1}{\sqrt{k}}\right)-I_{0}\left(\frac{r}{\sqrt{k}}\right)\right)\right] \tilde{\nabla}^{2} \phi_{22} \\
& \quad+\frac{2 i \alpha \operatorname{Re} \bar{k}}{r}\left(-\frac{1}{k} I_{0}\left(\frac{r}{\sqrt{k}}\right) r+\frac{2}{\sqrt{k}} I_{1}\left(\frac{r}{\sqrt{k}}\right)\right) \phi_{22} \\
& \quad+\frac{i \alpha \operatorname{Re}}{r}\left[\phi_{1} \phi_{1}^{\prime \prime \prime}-\phi_{1}^{\prime} \phi_{1}^{\prime \prime}-\frac{3}{r} \phi_{1} \phi_{1}^{\prime \prime}+\frac{1}{r}\left(\phi_{1}^{\prime}\right)^{2}+\frac{3}{r^{2}} \phi_{1} \phi_{1}^{\prime}+\frac{2}{r} \alpha^{2}\left(\phi_{1}\right)^{2}\right],
\end{aligned}
$$

with

$$
\begin{aligned}
& \overline{\bar{\nabla}}^{2}=\frac{d^{2}}{d r^{2}}-\frac{1}{r} \frac{d}{d r}, \quad \tilde{\tilde{\nabla}}^{2}=\frac{d^{2}}{d r^{2}}-\frac{1}{r} \frac{d}{d r}-4 \alpha^{2}, \\
& \phi_{20}^{\prime}(1)+\frac{1}{2}\left[\phi_{1}^{\prime \prime}(1)+\phi_{1}^{*^{\prime \prime}}(1)\right]+\frac{1}{4} \bar{k}\left[-\frac{1}{\sqrt{k}} I_{1}\left(\frac{1}{\sqrt{k}}\right)-\frac{1}{k} I_{0}\left(\frac{1}{\sqrt{k}}\right)\right]=0, \\
& \phi_{22}^{\prime}(1)+\frac{1}{2} \phi_{1}^{\prime \prime}(1)+\frac{1}{8} \bar{k}\left[-\frac{1}{\sqrt{k}} I_{1}\left(\frac{1}{\sqrt{k}}\right)-\frac{1}{k} I_{0}\left(\frac{1}{\sqrt{k}}\right)\right]=0, \\
& \phi_{22}(1)+\frac{1}{4} \phi_{1}^{\prime}(1)=0 .
\end{aligned}
$$

Thus, we obtained a set of differential equations together with the corresponding boundary conditions which are sufficient to determine the solution of the problem up to the second order in $\epsilon$.

Now, our main purpose is to find out solutions of differential equations for $\phi_{1}$, although equation (3.17) for $\phi_{1}$ is the fourth-order ordinary differential equation with variable coefficients. However, we can restrict our investigation to the case of pumping of an initially stagnant fluid, which is not subject to imposed pressure gradient. Thus, in this case $(\partial P / \partial z)_{0}=0$, which means constant $\bar{k}$ vanishes and we would be able to obtain a simple closed form analytical solution of this interesting case of free pumping. 
4. Free pumping (originally stationary fluid). Let us consider the case in which the pressure gradient $(\partial P / \partial z)_{0}$ vanishes. In this case, there will be no flow if the wall motion stops. Hence $\bar{k}=0$, then (3.17) becomes

$$
\left(\frac{d^{2}}{d r^{2}}-\frac{1}{r} \frac{d}{d r}-\beta^{2}\right)\left(\frac{d^{2}}{d r^{2}}-\frac{1}{r} \frac{d}{d r}-\alpha^{2}\right) \phi_{1}=0,
$$

in which $\beta^{2}=\alpha^{2}-i \alpha \operatorname{Re}-1 / k$ and the boundary conditions (3.18) with $\bar{k}=0$ becomes

$$
\phi_{1}^{\prime}(1)=0, \quad \phi_{1}(1)=-\frac{1}{2} .
$$

The boundary condition (4.2), together with the condition that the velocity must remain finite at $r=0$, then lead to the solution

$$
\phi_{1}(r)=c_{1} r I_{1}(\beta r)+c_{3} r I_{1}(\alpha r)
$$

where

$$
\begin{gathered}
c_{1}=\frac{-\alpha I_{0}(\alpha)}{2\left(\alpha I_{0}(\alpha) I_{1}(\beta)-\beta I_{0}(\beta) I_{1}(\alpha)\right)}, \\
c_{3}=\frac{-\beta I_{0}(\beta)}{2\left(\alpha I_{0}(\alpha) I_{1}(\beta)-\beta I_{0}(\beta) I_{1}(\alpha)\right)} .
\end{gathered}
$$

On substituting (4.3) and its conjugate into (3.20) and (3.23), we obtain

$$
\begin{aligned}
& \begin{aligned}
& \bar{\nabla}^{2}\left(\overline{\bar{\nabla}}^{2}-\frac{1}{k}\right) \phi_{20} \\
&=-\alpha^{2} \operatorname{Re}^{2}[ 2 c_{1} c_{1}^{*}\left[r \beta^{*} I_{1}(\beta r) I_{0}\left(\beta^{*} r\right)+r \beta I_{0}(\beta r) I_{1}\left(\beta^{*} r\right)-2 I_{1}(\beta r) I_{1}\left(\beta^{*} r\right)\right] \\
&+c_{1} c_{3}^{*}\left[r \beta I_{0}(\beta r) I_{1}(\alpha r)+r \alpha I_{1}(\beta r) I_{0}(\alpha r)-2 I_{1}(\beta r) I_{1}(\alpha r)\right] \\
&\left.+c_{1}^{*} c_{3}\left[r \beta^{*} I_{1}(\alpha r) I_{0}\left(\beta^{*} r\right)+r \alpha I_{0}(\alpha r) I_{1}\left(\beta^{*} r\right)-2 I_{1}\left(\beta^{*} r\right) I_{1}(\alpha r)\right]\right], \\
& \phi_{20}^{\prime}(1)=-\frac{1}{2}[ c_{1} \beta^{2} I_{1}(\beta)+\left(c_{3}+c_{3}^{*}\right) \alpha^{2} I_{1}(\alpha)+c_{1}^{*} \beta^{* 2} I_{1}\left(\beta^{*}\right) \\
&\left.+c_{1} \beta I_{0}(\beta)+c_{1}^{*} \beta^{*} I_{0}\left(\beta^{*}\right)+\left(c_{3}+c_{3}^{*}\right) \alpha I_{0}(\alpha)\right]=D .
\end{aligned}
\end{aligned}
$$

The right-hand side of (4.5) is a complicated function of $r$. We evaluate the righthand side numerically and represent the result approximately by a polynomial of the following form:

$$
\overline{\bar{\nabla}}^{2}\left(\overline{\bar{\nabla}}^{2}-\frac{1}{k}\right) \phi_{20}=-\alpha^{2} \operatorname{Re}^{2} \sum_{i=1}^{S} B_{i} r^{2 i},
$$

for different values of $\alpha$ and Re different $B_{i}$ must be used. Solving (4.7), we obtain

$$
\phi_{20}^{\prime}=L_{1}+\frac{i}{\sqrt{k}} L_{2} I_{0}\left(\frac{r}{\sqrt{k}}\right)-\alpha^{2} \operatorname{Re}^{2} \sum_{i=1}^{S} \frac{B_{i} r^{2 i+1}}{2 i(2 i+2)^{2}},
$$

where $L_{1}$ and $L_{2}$ are constants. 
Defining the following function:

$$
G(r)=\sum_{i=1}^{S} \frac{B_{i} r^{2 i+1}}{2 i(2 i+2)^{2}},
$$

we can write the boundary condition (4.6) in the form

$$
\phi_{20}^{\prime}(1)=D=L_{1}+\frac{i}{\sqrt{k}} L_{2} I_{0}\left(\frac{1}{\sqrt{k}}\right)-\alpha^{2} \operatorname{Re}^{2} G(1)
$$

on substituting (4.9) and (4.10) into (4.8), we get

$$
\phi_{20}^{\prime}(r)=\left[\alpha^{2} \operatorname{Re}^{2} G(1)+D\right] r+\frac{i}{\sqrt{k}} L_{2}\left(I_{0}\left(\frac{r}{\sqrt{k}}\right)-I_{0}\left(\frac{1}{\sqrt{k}}\right)\right) r-\alpha^{2} \operatorname{Re}^{2} G(r),
$$

if each term of (3.1), (3.2), (3.16), (3.19), and (4.3) is time-averaged over one period, we obtain the mean pressure gradient

$$
\begin{aligned}
\left(\frac{\bar{\partial} p}{\partial z}\right)_{2}= & \frac{1}{r \operatorname{Re}}\left[-\phi_{20}^{\prime \prime \prime}+\frac{1}{r} \phi_{20}^{\prime \prime}-\left(\frac{1}{r^{2}}-\frac{1}{k}\right) \phi_{20}^{\prime}\right] \\
& +\frac{i \alpha}{r^{2}}\left[\phi_{1} \phi_{1}^{*^{\prime \prime}}-\phi_{1}^{*} \phi_{1}^{\prime \prime}-\frac{1}{r}\left(\phi_{1} \phi_{1}^{*^{\prime}}-\phi_{1}^{*} \phi_{1}^{\prime}\right)\right]+O\left(\epsilon^{3}\right)
\end{aligned}
$$

substitution of (4.11) into (4.12) yields:

$$
\begin{aligned}
\left(\frac{\bar{\partial} p}{\partial z}\right)_{2}=-\alpha^{2} \operatorname{Re}[ & -\frac{G^{\prime \prime}(r)}{r}+\frac{1}{r^{2}} G^{\prime}(r)-\frac{1}{r^{3}} G(r)+\frac{1}{k r} G(r) \\
& -2 c_{1} c_{1}^{*} I_{1}(\beta r) I_{1}\left(\beta^{*} r\right)-c_{1} c_{3}^{*} I_{1}(\beta r) I_{1}(\alpha r) \\
& \left.-c_{1}^{*} c_{3} I_{1}(\alpha r) I_{1}\left(\beta^{*} r\right)\right]+\frac{H}{\mathrm{Re}}=Z+\frac{H}{\mathrm{Re}} .
\end{aligned}
$$

The time-averaged pressure gradient is not constant across the tube, but has a perturbation which varies with the radius. This perturbation function, which is denoted by $Z$, is the expression enclosed in the brackets in (4.13).

The solution for the mean axial velocity (averaged over time) is

$$
\begin{aligned}
\bar{v}_{z} & =-\frac{1}{r} \epsilon^{2} \phi_{20}^{\prime} \\
& =\epsilon^{2}\left[-\alpha^{2} \operatorname{Re}^{2}\left[G(1)-\frac{G(r)}{r}\right]-D+k \operatorname{Re}\left(\frac{\partial P}{\partial z}\right)_{2} \frac{\left[I_{0}(1 / \sqrt{k})-I_{0}(r / \sqrt{k})\right]}{2 I_{0}(1 / \sqrt{k})-1}\right] .
\end{aligned}
$$

From (4.14) a "critical reflux condition" can be defined as the condition $\bar{v}_{z}=0$ at $r=0$ (i.e., at the centre of the tube) using (4.13), this condition is

$$
\left(\frac{\partial \bar{P}}{\partial z}\right)_{2 c . r}=\frac{H}{\operatorname{Re}},
$$

where

$$
H=\frac{\left[2 I_{0}(1 / \sqrt{k})-1\right]}{k\left[I_{0}(1 / \sqrt{k})-1\right]}\left(\alpha^{2} \operatorname{Re}^{2} G(1)+D\right) .
$$


5. Stokes approximation. As Re $\rightarrow 0$ (the Reynolds number is considered so small that the inertia terms in the governing equations are negligible).

Equation (4.8) becomes

$$
\phi_{20}^{\prime}(r)=L_{1}+\frac{i}{\sqrt{k}} L_{2} I_{0}\left(\frac{r}{\sqrt{k}}\right)
$$

we can write the boundary condition equation (4.6) in the form

$$
\phi_{20}^{\prime}(1)=D=L_{1}+\frac{i}{\sqrt{k}} L_{2} I_{0}\left(\frac{1}{\sqrt{k}}\right),
$$

on substituting (5.2) into (5.1) we get

$$
\phi_{20}^{\prime}(r)=\left[D+\frac{i}{\sqrt{k}} L_{2}\left(I_{0}\left(\frac{r}{\sqrt{k}}\right)-I_{0}\left(\frac{1}{\sqrt{k}}\right)\right)\right] r,
$$

the solution for the mean-axial velocity is

$$
\bar{v}_{z}=-\frac{1}{r} \epsilon^{2} \phi_{20}^{\prime}(r)=-\epsilon^{2}\left[D+\frac{i}{\sqrt{k}} L_{2}\left(I_{0}\left(\frac{r}{\sqrt{k}}\right)-I_{0}\left(\frac{1}{\sqrt{k}}\right)\right)\right],
$$

where

$$
L_{2}=\left(\frac{-i \sqrt{k} D}{I_{0}(1 / \sqrt{k})-1}\right)
$$

6. Numerical results and discussion. In order to study the behavior of the solution, numerical calculations for several values of $\alpha, k$, and Re were carried out. Equation (4.14) shows that the mean axial velocity is dominated by the $D$ term and the parabolic term

$$
k \operatorname{Re}\left(\frac{\partial P}{\partial z}\right)_{2}\left(\frac{I_{0}(1 / \sqrt{k})-I_{0}(r / \sqrt{k})}{2 I_{0}(1 / \sqrt{k})-1}\right) .
$$

The constant $D$, which initially a rose from the non-slip conditions of the axial velocity on the wall and is related to the mean-velocity at the boundary of the tube (at $r=1$ ) by $\bar{v}_{z}=\epsilon^{2} D$. The parabolic term is due to the time-averaged second order pressure gradient set up by the peristaltic motion. In addition to the two terms mentioned above, the velocity has a perturbation term which is a function of $r$, given by

$$
\left(G(1)-\frac{G(r)}{r}\right)
$$

The variation of $D$ with $\alpha$ for various values of Re and $k$ is depicted in Figures 6.1, 6.2, and 6.3. The numerical results show that $D$ decreases with increasing Re and increases with increasing $k$.

The effects of $k$ and $(\partial P / \partial z)_{2}$ on the mean-velocity distribution and reversal flow are displayed in Figures 6.4-6.11. The results reveal that the mean-velocity distribution increases with increasing the permeability parameter $k$ and $(\partial P / \partial z)_{2}$. We notice that 


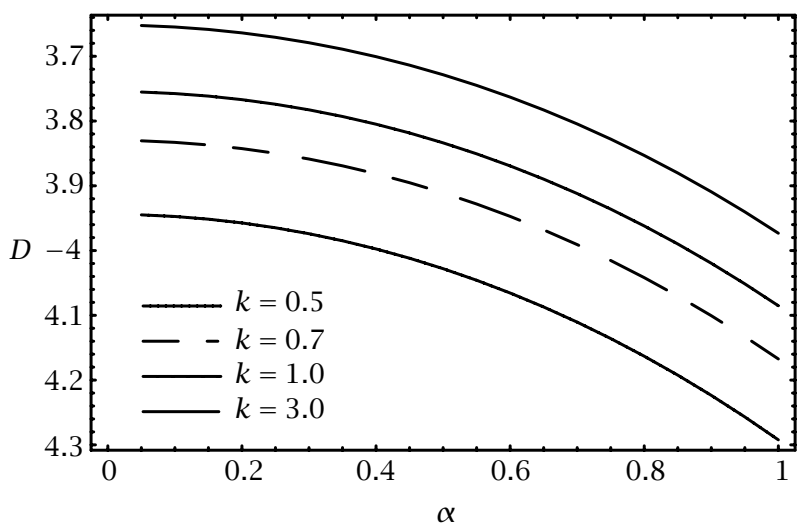

FIGURE 6.1. Variation of $D$ with wave number $\alpha$ and permeability parameter $k$ at $\operatorname{Re}=1.0$.

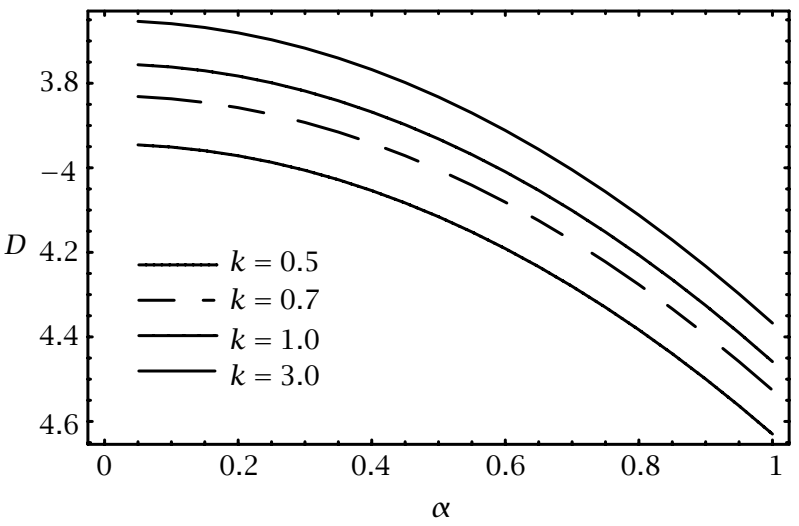

FIGURE 6.2. Variation of $D$ with wave number $\alpha$ and permeability parameter $k$ at $\mathrm{Re}=10.0$.

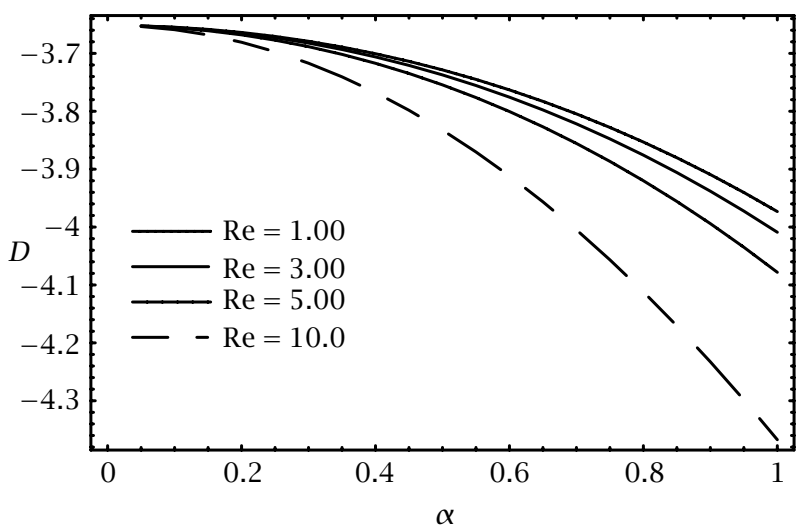

FIGURE 6.3. Variation of $D$ with wave number $\alpha$ and Reynolds number Re at $k=0.5$. 


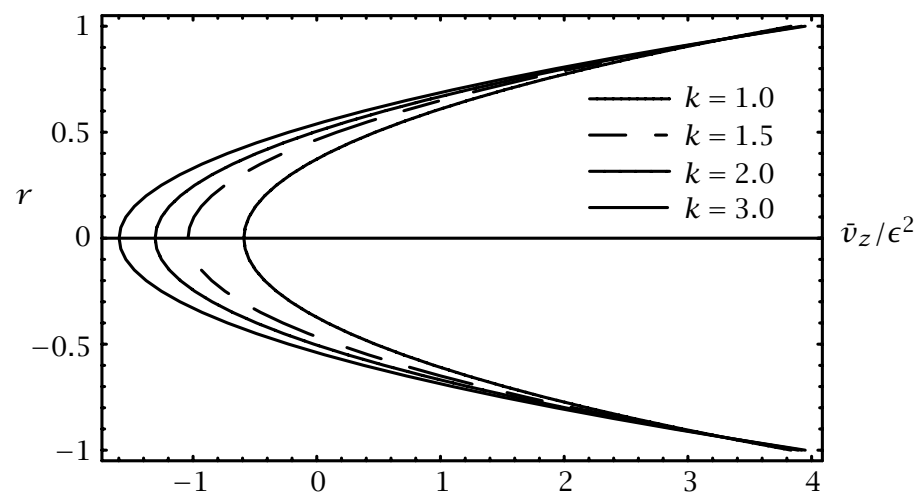

FIGURE 6.4. The effect of the permeability parameter $k$ on the mean-velocity distribution and reversal flow at $\operatorname{Re}=5.0,(\partial P / \partial z)_{2}=-5$, and $\alpha=0.1$.

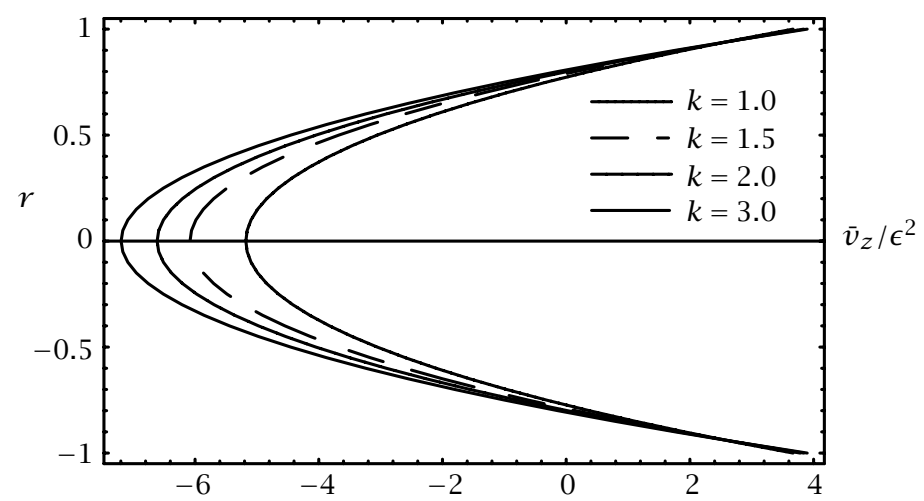

FIGURE 6.5. The effect of the permeability parameter $k$ on the mean-velocity distribution and reversal flow at $\operatorname{Re}=10.0,(\partial P / \partial z)_{2}=-5$, and $\alpha=0.1$.

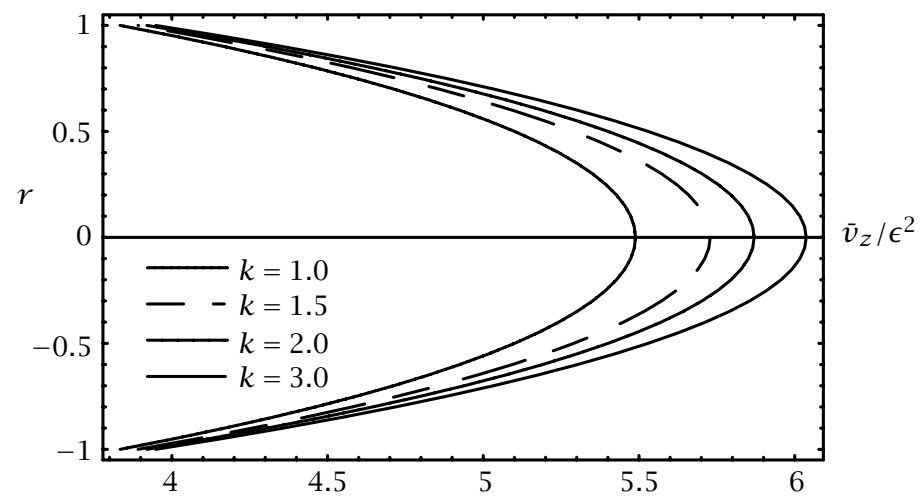

FIGURE 6.6. The effect of the permeability parameter $k$ on the mean-velocity distribution and reversal flow at $\operatorname{Re}=5.0,(\partial P / \partial z)_{2}=2.0$, and $\alpha=0.1$. 


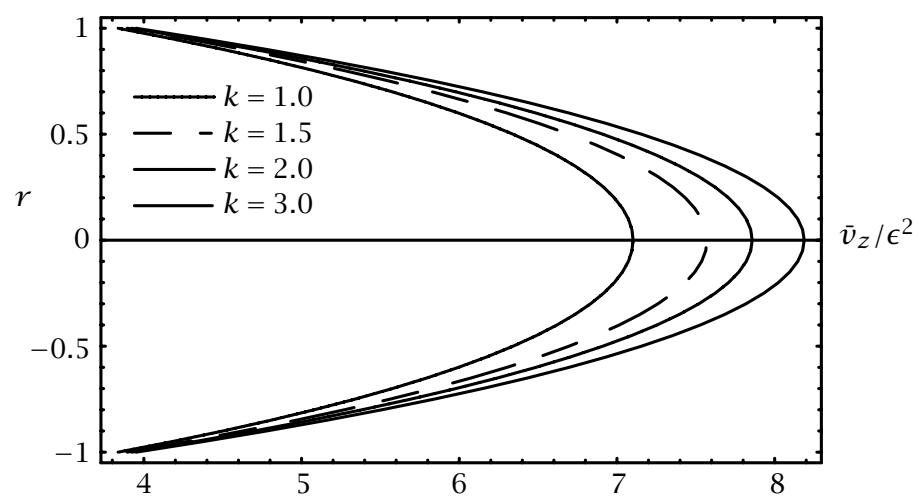

FIGURE 6.7. The effect of the permeability parameter $k$ on the mean-velocity distribution and reversal flow at $\operatorname{Re}=10.0,(\partial P / \partial z)_{2}=2.0$, and $\alpha=0.1$.

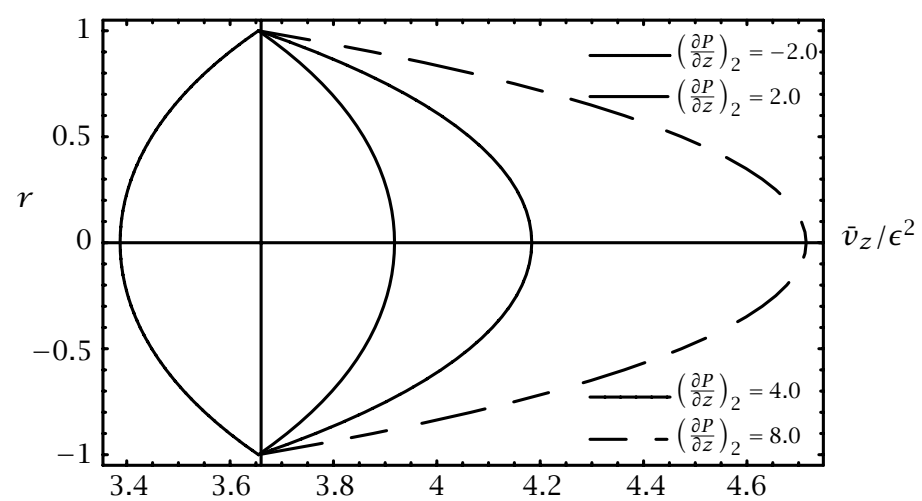

FIGURE 6.8. The effect of the mean second-order pressure gradient $(\partial P / \partial z)_{2}$ on the mean-velocity distribution and reversal flow at $\operatorname{Re}=1.0, k=0.5$, and $\alpha=0.1$.

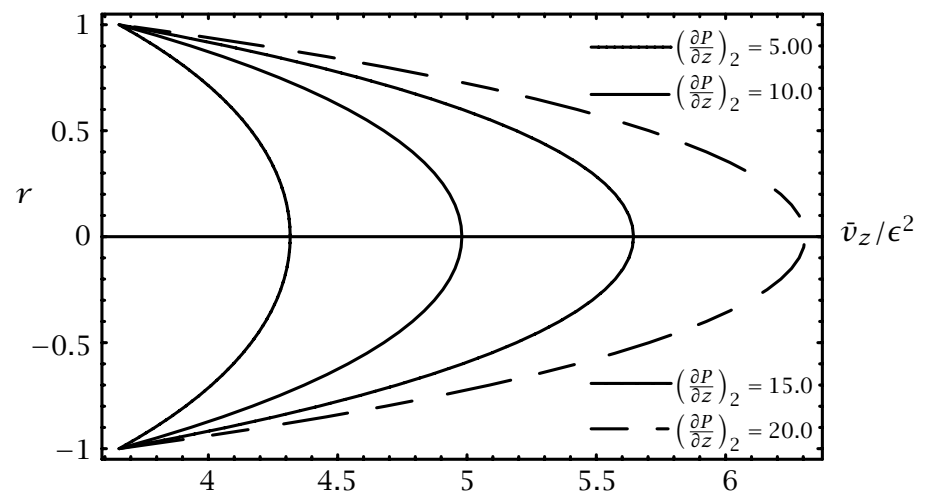

FIGURE 6.9. The effect of the mean second-order pressure gradient $(\partial P / \partial z)_{2}$ on the mean-velocity distribution and reversal flow at $\operatorname{Re}=1.0, k=0.5$, and $\alpha=0.1$. 


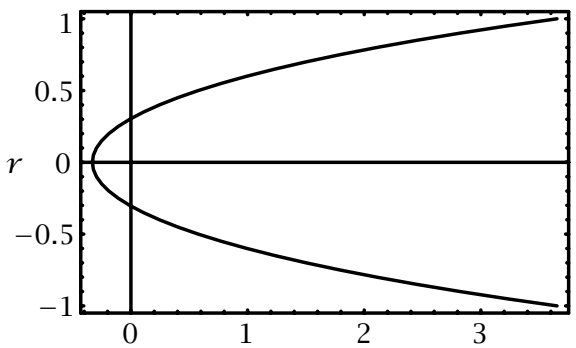

(a) $k=0.5$.

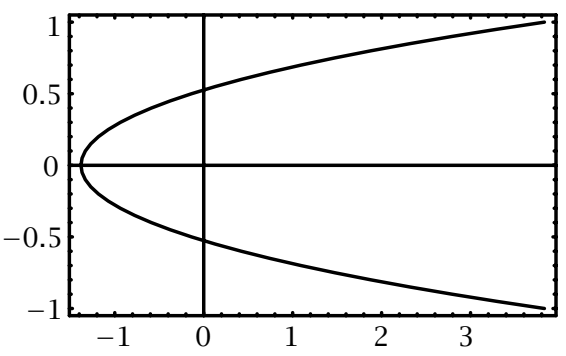

(c) $k=1.0$.

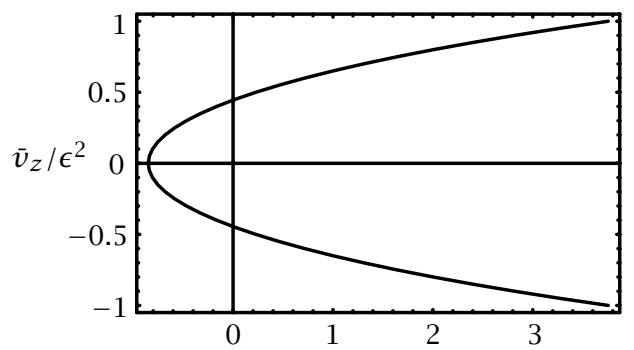

(b) $k=0.7$.

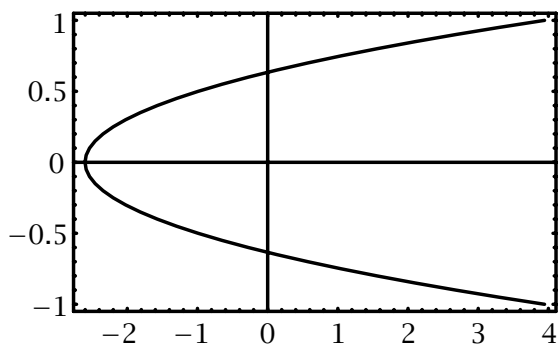

(d) $k=3.0$.

FIGURE 6.10. The effect of the permeability parameter $k$ on the meanvelocity distribution and reversal flow at $\operatorname{Re}=1.0,(\partial P / \partial z)_{2}=-10.0$, and $\alpha=0.1$.

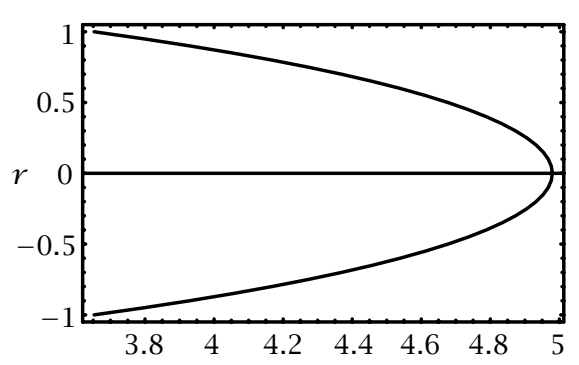

(a) $k=0.5$.

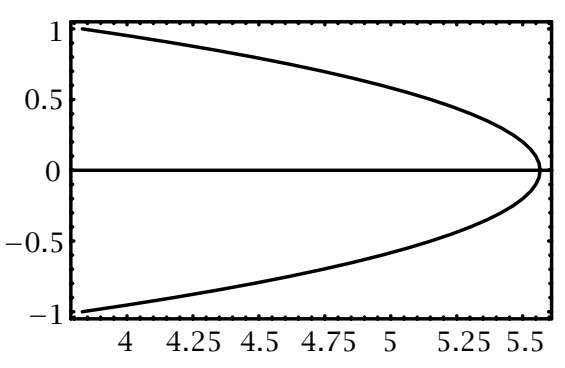

(c) $k=1.0$.

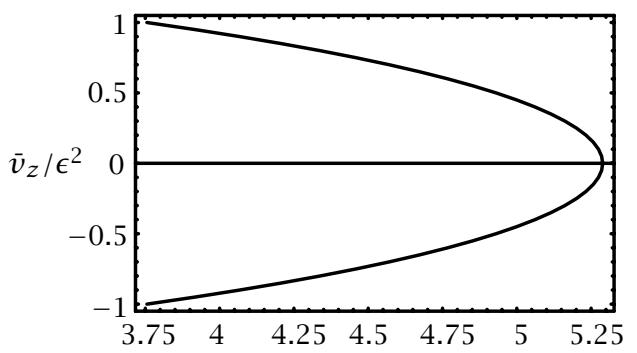

(b) $k=0.7$.

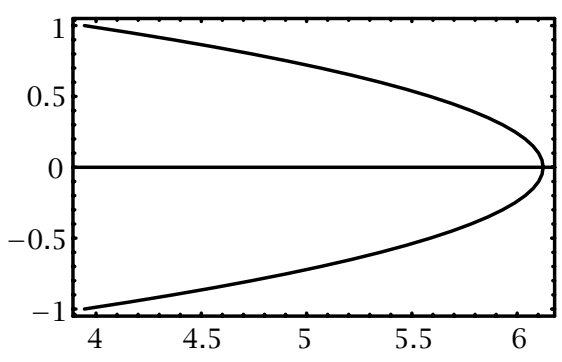

(d) $k=3.0$.

FIGURE 6.11. The effect of the permeability parameter $k$ on the meanvelocity distribution and reversal flow at $\operatorname{Re}=1.0,(\partial P / \partial z)_{2}=10.0$, and $\alpha=0.1$. 
as $k$ increases the velocity increases forward for $(\partial P / \partial z)_{2}>0$, while for $(\partial P / \partial z)_{2}<0$ the velocity increases backward (increasing the flux flow).

For $(\partial P / \partial z)_{2}<(\partial P / \partial z)_{2 c . r}$ there is no reflux and if $(\partial P / \partial z)_{2}>(\partial P / \partial z)_{2 c . r}$, there will be reflux and backward flow in the neighborhood of the centre line occurs.

\section{REFERENCES}

[1] A. M. El Misery, E. F. El Shehawey, and A. A. Hakeem, Peristaltic motion of an incompressible generalized Newtonian fluid in a planar channel, J. Phys. Soc. Jpn. 65 (1996), no. 11, 3524-3529.

[2] M. F. El Sayed, Electrohydrodynamic instability of two superposed viscous streaming fluids through porous medium, Can. J. Phys. 75 (1997), 499-508.

[3] E. F. El Shehawey, K. S. Mekheimer, S. F. Kaldas, and N. A. S. Afifi, Peristaltic transport through a porous medium, J. Biomath. 14 (1999), no. 1.

[4] M. Y. Jaffrin and A. H. Shapiro, Peristaltic pumping, Annual Review of Fluid Mechanics (Palo Alto, California, Palo Alto Publications), vol. 3, Annual Review, 1971, p. 13.

[5] T. W. Latham, Fluid motion in a peristaltic pumping, Master's thesis, Massachusetts, MIT Cambridge, 1966.

[6] K. S. Mekheimer, E. F. El Shehawey, and A. M. Elaw, Peristaltic motion of a particle-fluid suspension in a planar channel, Int. J. Theor. Phys. 37 (1998), no. 11, 2895-2920. Zbl 991.27313.

[7] D. A. Nield and A. Bejan, Convection in Porous Media, Springer-Verlag, New York, 1999. MR 2000b:76107. Zbl 924.76001.

[8] A. Raptis, N. Kafousias, and C. Z. Massalas, Free convection flow through a porous medium bounded by a vertical surface, Z. Angew. Math. Mech. (ZAMM) 62 (1982), 489.

[9] A. Raptis, C. Peridikis, and G. Tzivanidis, Free convection flow through a porous medium bounded by a vertical surface, J. Phys. D. Appl. Phys. 14 (1981), L99.

[10] R. K. Rathy, An Introduction to Fluid Dynamics, Oxford and IBH Publishing Co., New Delhi, 1976. MR 56\#17393.

[11] M. Saxena and V. P. Srivastava, Particulate suspension flow induced by sinusoidal peristaltic waves, J. Appl. Phys. 36 (1997), 386 (Japanese).

[12] A. E. Scheidegger, The Physics of Flow through Porous Media, McGraw-Hill, New York, 1963.

[13] V. P. Srivastava and L. M. Srivastava, Casson II, J. Biomech. 17 (1984), 821.

[14] _ Effects of Poiseuille flow on peristaltic transport of a particulate suspension, Z. Angew. Math. Phys. 46 (1995), no. 5, 655-679. MR 96f:76086. Zbl 836.76106.

[15] C. L. Varshney, Fluctuating flow of viscous fluid through a porous medium bounded by a porous plate., Indian J. Pure Appl. Math. 10 (1979), 1558-1564. Zbl 416.76049.

[16] K. Yamamoto and N. Iwamura, Flow with convective acceleration through a porous medium, J. Eng. Math. 10 (1976), 41-54. Zbl 376.76066.

[17] C. S. Yih and Y. C. Fung, Peristaltic waves in circular cylindrical tubes, J. Appl. Mech. 579 (1969), no. 36, 579-587.

Elsayed F. El Shehawey and Wahed El Sebaei: Department of Mathematics, Faculty of Education, Ain Shams University, Heliopolis, CAIro, EgYPT 


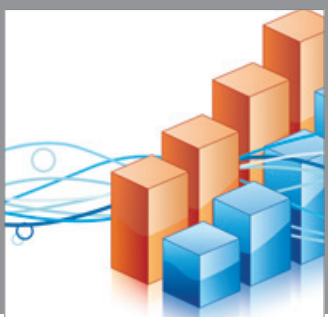

Advances in

Operations Research

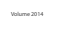

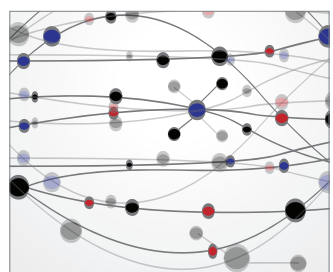

\section{The Scientific} World Journal
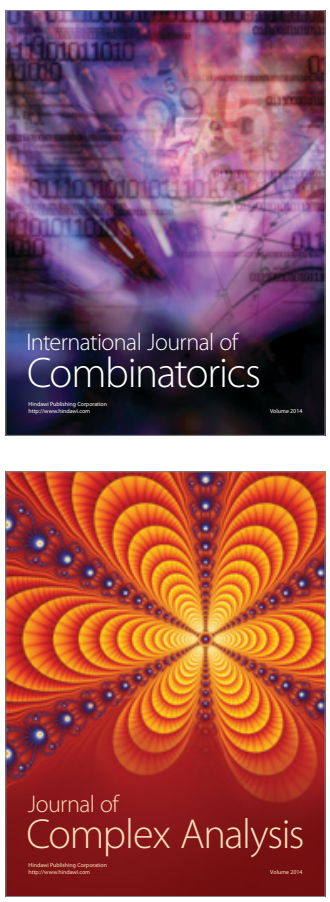

International Journal of

Mathematics and

Mathematical

Sciences
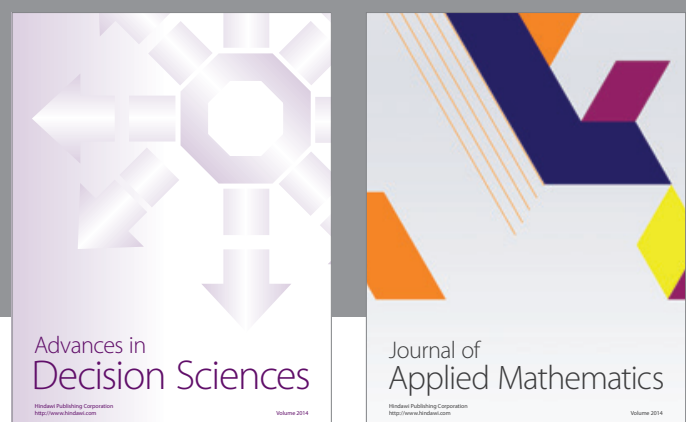

Journal of

Applied Mathematics
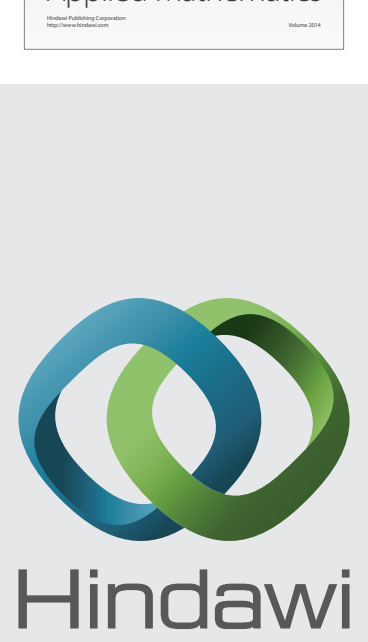

Submit your manuscripts at http://www.hindawi.com
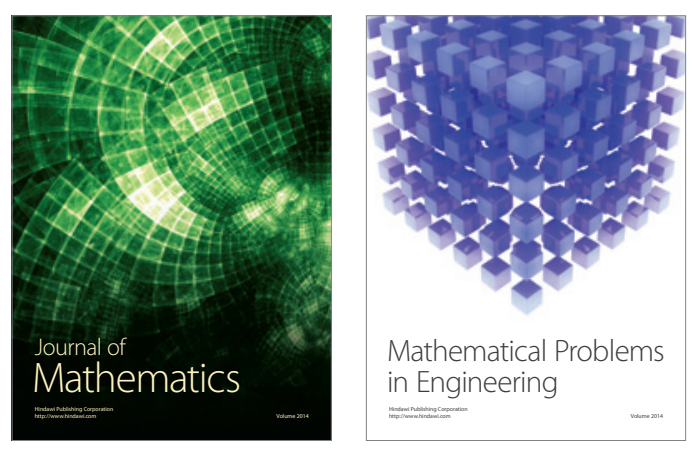

Mathematical Problems in Engineering
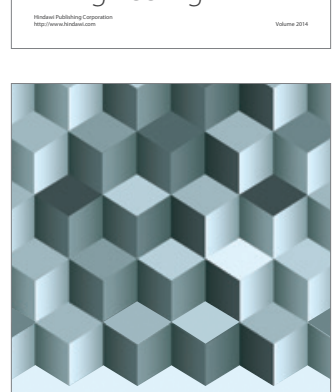

Journal of

Function Spaces
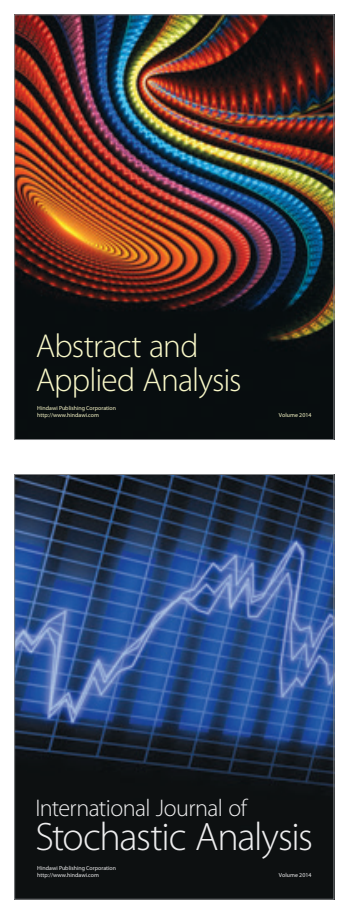

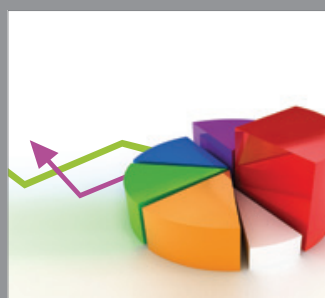

ournal of

Probability and Statistics

Promensencen
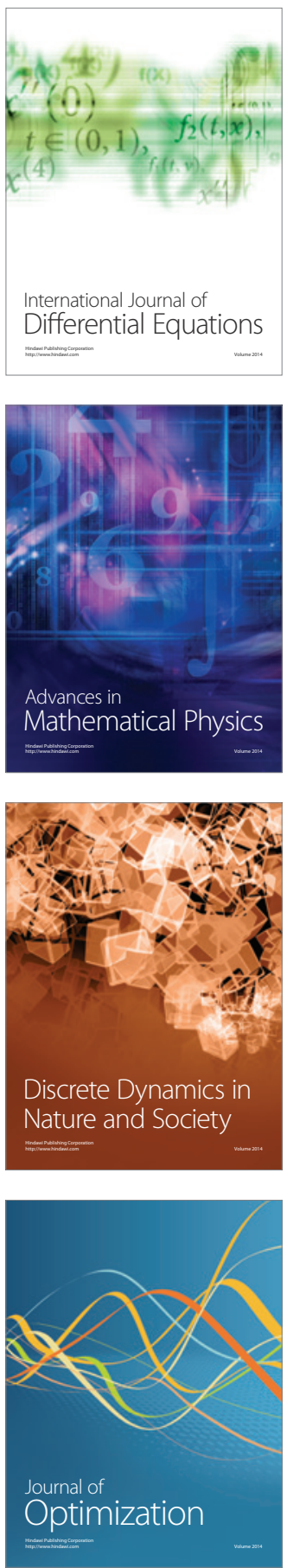